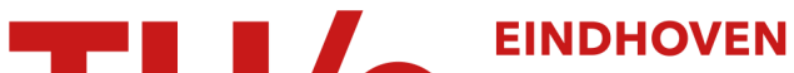 \\ UNIVERSITY OF \\ TECHNOLOGY
}

\section{Enhancing master-slave synchronization}

\section{Citation for published version (APA):}

Pena Ramirez, J., Arellano-Delgado, A., \& Nijmeijer, H. (2018). Enhancing master-slave synchronization: the effect of using a dynamic coupling. Physical Review E, 98(1), [012208].

https://doi.org/10.1103/PhysRevE.98.012208

DOI:

10.1103/PhysRevE.98.012208

Document status and date:

Published: 13/07/2018

\section{Document Version:}

Publisher's PDF, also known as Version of Record (includes final page, issue and volume numbers)

\section{Please check the document version of this publication:}

- A submitted manuscript is the version of the article upon submission and before peer-review. There can be important differences between the submitted version and the official published version of record. People interested in the research are advised to contact the author for the final version of the publication, or visit the $\mathrm{DOI}$ to the publisher's website.

- The final author version and the galley proof are versions of the publication after peer review.

- The final published version features the final layout of the paper including the volume, issue and page numbers.

Link to publication

\section{General rights}

Copyright and moral rights for the publications made accessible in the public portal are retained by the authors and/or other copyright owners and it is a condition of accessing publications that users recognise and abide by the legal requirements associated with these rights.

- Users may download and print one copy of any publication from the public portal for the purpose of private study or research.

- You may not further distribute the material or use it for any profit-making activity or commercial gain

- You may freely distribute the URL identifying the publication in the public portal.

If the publication is distributed under the terms of Article 25fa of the Dutch Copyright Act, indicated by the "Taverne" license above, please follow below link for the End User Agreement:

www.tue.nl/taverne

Take down policy

If you believe that this document breaches copyright please contact us at:

openaccess@tue.nl

providing details and we will investigate your claim. 


\title{
Enhancing master-slave synchronization: The effect of using a dynamic coupling
}

\author{
J. Pena Ramirez, ${ }^{1, *}$ A. Arellano-Delgado, ${ }^{2, \dagger}$ and H. Nijmeijer ${ }^{3, \dagger}$ \\ ${ }^{1}$ Applied Physics Division, Center for Scientific Research and Higher Education at Ensenada (CONACYT-CICESE). \\ Carretera Ensenada-Tijuana 3918, Zona Playitas, Ensenada, Codigo Postal 22860, Baja California, Mexico \\ ${ }^{2}$ Engineering, Architecture, and Design Faculty, Autonomous University of Baja California (CONACYT-UABC). \\ Carretera Ensenada-Tijuana 3917, Zona Playitas, Ensenada, Codigo Postal 22860, Baja California, Mexico \\ ${ }^{3}$ Department of Mechanical Engineering, Eindhoven University of Technology. P.O. Box 513, 5600 MB Eindhoven, The Netherlands
}

(Received 26 May 2017; revised manuscript received 27 April 2018; published 13 July 2018)

\begin{abstract}
This paper introduces a modified master-slave synchronization scheme for dynamical systems. In contrast to the standard configuration, the slave system does not receive any driving signal from the master, but rather the interaction is through a linear dynamical system. The key feature of the proposed coupling scheme is that it induces synchronization in certain systems that cannot be synchronized when using the classical static interconnection. Likewise, the dynamic coupling achieves synchronization for arbitrarily large coupling strength values in certain systems for which the classical configuration is applicable only within a narrow interval of coupling strength values. The performance of the synchronization scheme is illustrated in pairs of identical chaotic and mechanical oscillators
\end{abstract}

DOI: 10.1103/PhysRevE.98.012208

\section{INTRODUCTION}

An intriguing phenomenon occurring in dynamical systems is synchronization. In particular, when two or more systems are allowed to interact, it may happen that they show "sympathy" for each other, i.e., they can adjust their rhythms.

One of the key elements for synchronization-perhaps the most important-is that there exists a communication channel, called coupling, through which the systems "communicate" in order to adjust their rhythms. In particular, in the literature of chaotic systems, there is a large collection of coupling schemes, such as, for example, the celebrated Pecora and Carroll configuration [1], the well-known master-slave configuration [2], diffusive coupling [3], mutual coupling [4], environmental coupling [5], dynamic coupling [6], transient uncoupling [7], just to mention a few.

Among these schemes, we want to draw attention to the standard master-slave configuration, described by [2]

$$
\begin{aligned}
\dot{\boldsymbol{x}}_{m} & =\boldsymbol{F}\left(\boldsymbol{x}_{m}\right), \\
\dot{\boldsymbol{x}}_{s} & =\boldsymbol{F}\left(\boldsymbol{x}_{s}\right)+k \boldsymbol{C}\left(\boldsymbol{x}_{m}-\boldsymbol{x}_{s}\right),
\end{aligned}
$$

where $\boldsymbol{x}_{m}$ and $\boldsymbol{x}_{s}$ are $n$-dimensional state vectors corresponding to the master and slave system, respectively, function $\boldsymbol{F}$ is, in general, a smooth and nonlinear function, $k$ is the coupling strength, and $\boldsymbol{C}$ is a suitably chosen coupling matrix.

This configuration has been extensively studied and used for inducing synchronization in a large class of dynamical systems, cf. Refs. [8-10]. In fact, if the systems are semipassive and

\footnotetext{
*Corresponding author: jpena@ cicese.mx

†adrian.arellano@uabc.edu.mx

†h.nijmeijer@tue.nl
}

convergent, synchronization will be achieved for "sufficiently large" coupling strength [11].

However, for certain systems, e.g., the Rössler system, this coupling scheme has the following well-known limitations: for certain coupling matrices $\boldsymbol{C}$, it is either impossible to induce synchronization or synchronization is observed only in a narrow interval of coupling strength values, cf. Ref. [12].

In a recent letter, it has been shown that the limitations on the values of coupling strength can be removed by transiently uncoupling the slave from the master system [7]. However, switching off the coupling at the wrong instant may result in loss of synchrony.

This paper presents a modified master-slave configuration in which the master and slave systems have an indirect interaction, i.e., they are not connected through common signals. Instead, the slave system receives a coupling signal, which is dynamically generated by a second order linear system.

It is demonstrated that by using a dynamic interconnection it is possible to induce synchronization in certain systems for which the classical master-slave scheme with static coupling may not be able to induce synchronization. Likewise, the proposed coupling scheme enforces synchronization for arbitrarily large coupling strength values in certain systems for which the static master-slave coupling has an upper bound on the value of coupling strength.

The stability of the synchronous solution in the coupled systems is investigated by using the master stability function approach [13]. In particular, a suitable variational equation is derived, and the corresponding largest transverse Lyapunov exponent is computed in order to obtain necessary conditions for the local stability of the synchronous solution. Likewise, the Lyapunov stability theory is used in order to tune the parameters in the dynamic coupling.

In the analysis, we consider as particular cases, a harmonic oscillator and several chaotic systems, including: the chaotic 


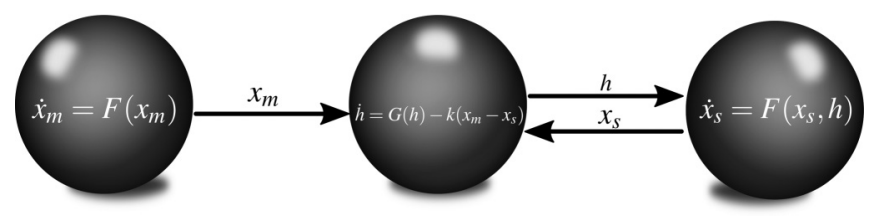

FIG. 1. Proposed dynamic master-slave configuration.

Rössler system, Chua's system, the Hindmarsh-Rose neuronal model, and the forced Duffing and van der Pol equations.

The results presented in this paper suggest that the onset of synchronization in the master-slave scheme is enhanced when the static coupling is replaced by a dynamic coupling.

The rest of the paper is organized as follows. Section II introduces the proposed synchronization scheme and the corresponding stability analysis. Then, some application examples are presented in Sec. III. Finally, a discussion and some conclusions are provided in Sec. IV.

\section{PROPOSED SYNCHRONIZATION SCHEME}

The proposed coupling scheme, see Fig. 1, is described by the set of equations,

$$
\begin{aligned}
\dot{\boldsymbol{x}}_{m} & =\boldsymbol{F}\left(\boldsymbol{x}_{m}\right), \\
\dot{\boldsymbol{x}}_{s} & =\boldsymbol{F}\left(\boldsymbol{x}_{s}\right)-\boldsymbol{B}_{1} \boldsymbol{h}, \\
\dot{\boldsymbol{h}} & =\boldsymbol{G} \boldsymbol{h}-k \boldsymbol{B}_{2}\left(\boldsymbol{x}_{m}-\boldsymbol{x}_{s}\right),
\end{aligned}
$$

where $\boldsymbol{x}_{m}, \boldsymbol{x}_{s} \in \mathbb{R}^{n}$ denote the state vectors of the master and slave, respectively, $\boldsymbol{h}=\left(h_{1}, h_{2}\right)^{T}$ with $h_{i} \in \mathbb{R}, i=1,2$ are the state variables of the dynamic coupling, the vector field $\boldsymbol{F}$ is assumed to be sufficiently smooth, it can be either linear or nonlinear, and $k$ is the coupling strength.

On the other hand, $\boldsymbol{B}_{1} \in \mathbb{R}^{n \times 2}$ and $\boldsymbol{B}_{2} \in \mathbb{R}^{2 \times n}$ are the coupling matrices. In this paper, it will be assumed that only one of the entries of matrix $B_{j}, j=1,2$ is equal to 1 and the remaining elements are 0 .

The assumption for $B_{2}$ indicates that only one element of the master is coupled to one element of the slave, see Eq. (5), whereas the assumption for $\boldsymbol{B}_{1}$ reveals that the dynamic coupling is applied to only one equation of the slave system, see Eq. (4).

The matrix $\boldsymbol{G}$ of the dynamic coupling given in Eq. (5), is defined by

$$
\boldsymbol{G}=\left[\begin{array}{cc}
-\alpha & 1 \\
-\gamma_{1} & -\gamma_{2}
\end{array}\right]
$$

where $\gamma_{1}, \gamma_{2}$, and $\alpha$ are positive constants to be chosen. Furthermore, note that the solutions of the dynamic coupling Eq. (5) vanish asymptotically when synchronization is achieved, i.e., when $\boldsymbol{x}_{m}=\boldsymbol{x}_{s}$.

The design of the coupling system, see Eqs. (5) and (6), has been inspired in the so-called Huygens' coupling, which in its simplest form, can be seen as a mass-spring-damper oscillator $[14,15]$. Note that, for $\alpha=k=0$, systems (5) and (6) are indeed the dynamics of a mass-spring-damper system with stiffness coefficient $\gamma_{1}$ and damping coefficient $\gamma_{2}$. We have also added the parameter $\alpha$ in (6), which allows for having more freedom in choosing $\gamma_{1}$ and $\gamma_{2}$.

\section{A. Stability analysis based on the master stability function}

Next, we introduce the tools, which are necessary for studying the onset of (stable) synchronous solutions in the proposed configuration described by Eqs. (3)-(5). These tools are based on the well-known master stability function approach [13].

First, it should be noted that the variational equation governing the behavior around the synchronous solution $\boldsymbol{\xi}:=\boldsymbol{x}_{m}-\boldsymbol{x}_{s}=0$ and $\boldsymbol{h}=0$ is

$$
\left[\begin{array}{l}
\dot{\boldsymbol{\xi}} \\
\dot{\boldsymbol{h}}
\end{array}\right]=\underbrace{\left[\begin{array}{cc}
\boldsymbol{D F}\left(\boldsymbol{x}_{m}\right) & \boldsymbol{B}_{1} \\
-k \boldsymbol{B}_{2} & \boldsymbol{G}
\end{array}\right]}_{\widetilde{\boldsymbol{D F}}\left(\boldsymbol{x}_{m}\right)}\left[\begin{array}{l}
\boldsymbol{\xi} \\
\boldsymbol{h}
\end{array}\right],
$$

where $\boldsymbol{D} \boldsymbol{F}=\partial_{\boldsymbol{x}_{m}} \boldsymbol{F}$ is the Jacobian of the vector function $\boldsymbol{F}$ evaluated on a (bounded) trajectory $\boldsymbol{x}_{m}$ of the master system.

The stability of the synchronous solution $\boldsymbol{e}:=(\boldsymbol{\xi}, \boldsymbol{h})=0$ can be investigated by looking at the largest (transverse) Lyapunov exponent $\lambda \stackrel{\perp}{\max }[16]$,

$$
\lambda \stackrel{\perp}{\perp}=\lim _{t \rightarrow \infty} \frac{\ln |\boldsymbol{e}(t)|-\ln |\boldsymbol{e}(0)|}{t},
$$

which is determined from Eq. (7).

If $\lambda_{\max }^{\perp}<0$, then the synchronous solution is (locally) stable, i.e., small disturbances will vanish. On the other hand, if $\lambda_{\max }^{\perp}>0$, the synchronous solution is unstable [16].

\section{B. Tuning the dynamic master-slave coupling}

The proposed coupling scheme requires tuning three parameters, namely, $\alpha, \gamma_{1}, \gamma_{2}$, see Eqs. (5) and (6). The first requirement for these parameters is that they should be positive. In this way, it is guaranteed that matrix $\boldsymbol{G}$ in Eq. (6) is Hurwitz and, consequently, when $\boldsymbol{x}_{m}=\boldsymbol{x}_{s}$ the coupling signal $\boldsymbol{h}(t)$, see Eq. (5), vanishes.

A second requirement that should be fulfilled when choosing the parameters of the dynamic coupling is the following. Assume that function $\boldsymbol{F}\left(\boldsymbol{x}_{i}\right)$ for $i=m, s$ can be separated into a linear and a nonlinear part, i.e., $\boldsymbol{F}\left(\boldsymbol{x}_{i}\right)=\boldsymbol{E} \boldsymbol{x}_{i}+\boldsymbol{f}\left(\boldsymbol{x}_{i}\right)$, where $\boldsymbol{E} \in \mathbb{R}^{n \times n}$ is a constant matrix and $\boldsymbol{f}\left(\boldsymbol{x}_{i}\right) \in \mathbb{R}^{n}$ is a nonlinear vector. Under this assumption and defining the synchronization error $\boldsymbol{e}_{p}:=\left(\boldsymbol{x}_{m}-\boldsymbol{x}_{s}, \boldsymbol{h}\right)^{T}$, the error dynamics for the proposed configuration, see Eqs. (3)-(5), is given by

$$
\dot{\boldsymbol{e}}_{p}=\boldsymbol{A}_{p} \boldsymbol{e}_{p}+\boldsymbol{g}_{p}\left(t, \boldsymbol{e}_{p}\right),
$$

where

$$
\boldsymbol{A}_{p}=\left[\begin{array}{cc}
\boldsymbol{E} & \boldsymbol{B}_{1} \\
-k \boldsymbol{B}_{2} & \boldsymbol{G}
\end{array}\right], \quad \boldsymbol{g}_{p}(\cdot)=\left[\begin{array}{c}
\boldsymbol{f}\left(\boldsymbol{x}_{m}\right)-\boldsymbol{f}\left(\boldsymbol{x}_{s}\right) \\
\boldsymbol{O}
\end{array}\right],
$$

and $\boldsymbol{O}=(0,0)^{T}$.

Furthermore, the "perturbation" term $\boldsymbol{g}_{p}\left(t, \boldsymbol{e}_{p}\right)$ is a vanishing perturbation [17] because on the synchronization manifold where $\boldsymbol{x}_{m}=\boldsymbol{x}_{s}$ it holds that $\boldsymbol{g}_{p}(t, 0)=0$ and $\left\|\boldsymbol{g}_{p}\left(t, \boldsymbol{e}_{p}\right)\right\| \leqslant$ $\gamma\left\|\boldsymbol{e}_{p}\right\|$ for certain $\gamma \in \mathbb{R}_{+}$where the notation $\|\cdot\|$ denotes a norm.

Then, using the stability theory for perturbed systems, see, e.g., Ref. [17], it follows that the parameters $\alpha, \gamma_{1}$, and $\gamma_{2}$ in 
Eq. (6) should be chosen such that matrix $\boldsymbol{A}_{p}$ in (9) is Hurwitz. Furthermore, this choice guarantees that system (9) is locally stable.

\section{APPLICATION EXAMPLES}

In this section, the proposed synchronization scheme presented in Eqs. (3)-(5) is used to synchronize pairs of identical oscillators for which the classical master-slave configuration, see Eqs. (1) and (2), either cannot induce synchronization or achieves synchronization in a very narrow interval of coupling strength values.

\section{A. Example 1: Synchronization of "nonsynchronizable" mechanical oscillators}

A simple but illuminating example showing the advantages of the the proposed coupling scheme over the traditional one is the harmonic oscillator. For this system, it is impossible to achieve synchronization by using the classical masterslave scheme. To show this, consider the classical configuration (1) and (2) with $\boldsymbol{x}_{i}=:\left(x_{1 i}, x_{2 i}\right)^{T}$ for $i=m, s, \boldsymbol{F}\left(\boldsymbol{x}_{i}\right)=$ $\boldsymbol{A} \boldsymbol{x}_{i}$, where $\boldsymbol{A}$ is a constant square matrix given by $\boldsymbol{A}:=$ $\left[(0,1),\left(-\omega^{2}, 0\right)\right]$ and $\omega \in \mathbb{R}_{+}$is the natural frequency of the uncoupled oscillators. The variables $x_{1 i}$ and $x_{2 i}$ denote the position and velocity of oscillator $i$ for $i=m, s$, respectively. Furthermore, assuming that the coupling between the master and the slave oscillators is through the position variable, the corresponding coupling matrix $\boldsymbol{C} \in \mathbb{R}^{2 \times 2}$, see Eq. (2), is defined as follows: $\boldsymbol{C}_{i j}=1$ for $i=2, j=1$, and $\boldsymbol{C}_{i j}=0$ otherwise.

Then, the pair of harmonic oscillators, interacting via the classical (static) master-slave scheme (1) and (2) can be written in the form

$$
\begin{aligned}
& \text { Master: }\left\{\begin{array}{l}
\dot{x}_{1 m}=x_{2 m}, \\
\dot{x}_{2 m}=-\omega^{2} x_{1 m},
\end{array}\right. \\
& \text { Slave: }\left\{\begin{array}{l}
\dot{x}_{1 s}=x_{2 s}, \\
\dot{x}_{2 s}=-\omega^{2} x_{1 s}+k\left(x_{1 m}-x_{1 s}\right) .
\end{array}\right.
\end{aligned}
$$

The global stability properties of the synchronous solution $\boldsymbol{x}_{m}=\boldsymbol{x}_{s}$ can be easily determined by using the theory of linear systems, see, e.g., Ref. [18]. As a first step, the following synchronization errors are defined: $e_{1}=x_{1 m}-x_{1 s}, e_{2}=$ $x_{2 m}-x_{2 s}$. The resulting error dynamics is then described by

$$
\dot{\boldsymbol{e}}=\boldsymbol{A}_{e} \boldsymbol{e},
$$

where $\boldsymbol{e}=\left(e_{1}, e_{2}\right)^{T}$ and

$$
\boldsymbol{A}_{e}=\left[\begin{array}{cc}
0 & 1 \\
-\left(\omega^{2}+k\right) & 0
\end{array}\right] .
$$

Next, the stability properties of the error dynamics are determined from the location of the eigenvalues of matrix $\boldsymbol{A}_{e}$, which are $\lambda_{1}=-\lambda_{2}=\sqrt{-\left(\omega^{2}+k\right)}$. Depending on the value of $k$, we will have the following three scenarios:

(a) For $k>-\omega^{2}$, the eigenvalues of $\boldsymbol{A}_{e}$ are complex conjugated (with zero real parts) and, consequently, the error dynamics is oscillatory,

(b) for $k=-\omega^{2}$, the eigenvalues of $\boldsymbol{A}_{e}$ are both zero, which indicates that the error dynamics is constant, and (c) for $k<-\omega^{2}$, the eigenvalues of $\boldsymbol{A}_{e}$ are real, one positive and one negative, which shows that for this $k$ the error dynamics is unstable.

In conclusion, it is impossible to find any value of $k \in \mathbb{R}$ such that the error dynamics are globally asymptotically stable. Therefore, the classical master-slave coupling fails to synchronize the oscillators.

On the other hand, the proposed dynamic coupling successfully induces synchronization in the coupled harmonic oscillators. To show this, consider Eqs. (3)-(5) with $\boldsymbol{x}_{i}$ and $\boldsymbol{F}\left(\boldsymbol{x}_{i}\right)$ as defined above. Furthermore, the coupling matrices $\boldsymbol{B}_{1}$ and $\boldsymbol{B}_{2}$ in (4) and (5) are chosen as follows:

$$
\boldsymbol{B}_{1}=\left[\begin{array}{ll}
0 & 0 \\
0 & 1
\end{array}\right] \text { and } \boldsymbol{B}_{2}=\left[\begin{array}{ll}
0 & 0 \\
1 & 0
\end{array}\right] \text {. }
$$

The choice of $\boldsymbol{B}_{2}$ follows from the assumption that only the position variable of the master and slave systems is used for driving the dynamic coupling (5). On the other hand, the choice of $\boldsymbol{B}_{2}$ indicates that the dynamic coupling signal $h_{2}$ is applied to the second equation of the slave system.

Then, the pair of harmonic oscillators interacting via the proposed dynamic master-slave scheme (3)-(5) takes the form

$$
\begin{gathered}
\text { Master: }\left\{\begin{array}{l}
\dot{x}_{1 m}=x_{2 m}, \\
\dot{x}_{2 m}=-\omega^{2} x_{1 m},
\end{array}\right. \\
\text { Slave: }\left\{\begin{array}{l}
\dot{x}_{1 s}=x_{2 s}, \\
\dot{x}_{2 s}=-\omega^{2} x_{1 s}-h_{2},
\end{array}\right. \\
\text { counamic } \\
\begin{array}{l}
\dot{h}_{1}=-\alpha h_{1}+h_{2}, \\
\dot{h}_{2}=-\gamma_{1} h_{1}-\gamma_{2} h_{2}-k\left(x_{1 m}-x_{1 s}\right) .
\end{array}
\end{gathered}
$$

By defining the synchronization errors $e_{1}=x_{1 m}-x_{1 s}$, $e_{2}=x_{2 m}-x_{2 s}, h_{1}=0$, and $h_{2}=0$, the stability of the synchronous solution can be determined from the error dynamics $\dot{\boldsymbol{e}}_{p}=\boldsymbol{A}_{p} \boldsymbol{e}_{p}$, see Eq. (9), where $\boldsymbol{e}_{p}=\left(e_{1}, e_{2}, h_{1}, h_{2}\right)^{T}$ and

$$
\boldsymbol{A}_{p}=\left[\begin{array}{cccc}
0 & 1 & 0 & 0 \\
-\omega^{2} & 0 & 0 & 1 \\
0 & 0 & -\alpha & 1 \\
-k & 0 & -\gamma_{1} & -\gamma_{2}
\end{array}\right]
$$

The characteristic polynomial of $\boldsymbol{A}_{p}$ is given by

$$
p(\lambda)=\lambda^{4}+a_{3} \lambda^{3}+a_{2} \lambda^{2}+a_{1} \lambda+a_{0},
$$

where $a_{3}=\alpha+\gamma_{2}, a_{2}=\gamma_{1}+\omega+\alpha \gamma_{2}, a_{1}=k+\omega\left(\alpha+\gamma_{2}\right)$, and $a_{0}=\alpha k+\omega\left(\gamma_{1}+\alpha \gamma_{2}\right)$. Hence, the synchronous solution will be globally asymptotically stable if and only if the following conditions are satisfied:

$$
\begin{aligned}
a_{3}, a_{0} & >0, \quad l=0, \\
\left(\alpha+\gamma_{2}\right)\left(\gamma_{1}+\alpha \gamma_{2}\right)-k & >0, \\
\left(\gamma_{1}-\omega\right)\left(\alpha+\gamma_{2}\right)-\alpha^{2} \gamma_{2}-\alpha^{3}-k & >0 .
\end{aligned}
$$

If, for example, we take $\alpha=0, \gamma_{1}=2 k+\omega$, and $\gamma_{2}=1$, then the above conditions are satisfied for all $k>0$, i.e., the synchronous solution is globally asymptotically stable for any (positive) coupling strength value.

This simple but illustrative example has shown that if the coupling is dynamic rather than static, then the master and slave oscillators can possibly achieve synchronization. 


\section{B. Example 2: Synchronization of nonsynchronizable chaotic systems}

This section presents another example for which the classical master-slave configuration fails to synchronize a pair of chaotic systems. For this, consider a pair of identical Rössler oscillators [19] coupled via the traditional master-slave configuration, i.e., Eqs. (1) and (2), with $\boldsymbol{x}_{m}=$ : $\left(x_{1 m}, x_{2 m}, x_{3 m}\right)^{T}, \boldsymbol{x}_{s}=:\left(x_{1 s}, x_{2 s}, x_{3 s}\right)^{T}$, and the nonlinear functions $\boldsymbol{F}\left(\boldsymbol{x}_{i}\right)=\left(\left(-y_{i}-z_{i}\right),\left(x_{i}+a y_{i}\right),\left(b+\left(x_{i}-c\right) z_{i}\right)\right)^{T}$ for $i=m, s$. Moreover, consider the case where the coupling is through only one component of the master and one component of the slave, namely $x_{2 m}$ and $x_{2 s}$, respectively. Likewise, consider the scenario when the coupling term is applied to the first equation of the slave system. Then, the coupling matrix $\boldsymbol{C} \in \mathbb{R}^{3 \times 3}$ is defined by $\boldsymbol{C}_{i j}=1$ for $i=1, j=2$, and $\boldsymbol{C}_{i j}=0$ otherwise.

Then, the pair of identical Rössler systems interacting via the classical master-slave configuration (1) and (2) is described by

$$
\begin{aligned}
& \text { Master: }\left\{\begin{array}{l}
\dot{x}_{1 m}=-x_{2 m}-x_{3 m}, \\
\dot{x}_{2 m}=x_{1 m}+a x_{2 m}, \\
\dot{x}_{3 m}=b+x_{1 m} x_{3 m}-c x_{3 m},
\end{array}\right. \\
& \text { Slave: }\left\{\begin{array}{l}
\dot{x}_{1 s}=-x_{2 s}-x_{3 s}+k\left(x_{2 m}-x_{2 s}\right), \\
\dot{x}_{2 s}=x_{1 s}+a x_{2 s}, \\
\dot{x}_{3 s}=b+x_{1 s} x_{3 s}-c x_{3 s},
\end{array}\right.
\end{aligned}
$$

In Ref. [12], it is demonstrated that it is impossible to achieve synchronization in the coupled Rössler oscillators (22) and (23).

In contrast, the dynamic master-slave configuration for the pair of Rössler systems can be written in the form of Eqs. (3)(5) by defining the state vectors $\boldsymbol{x}_{m}, \boldsymbol{x}_{s}$ and the nonlinear functions $\boldsymbol{F}\left(\boldsymbol{x}_{i}\right), i=m, s$ as above. The coupling matrices $\boldsymbol{B}_{1}$ and $\boldsymbol{B}_{2}$ are chosen as follows:

$$
\boldsymbol{B}_{1}=\left[\begin{array}{ll}
0 & 1 \\
0 & 0 \\
0 & 0
\end{array}\right] \text { and } \boldsymbol{B}_{2}=\left[\begin{array}{ccc}
0 & 0 & 0 \\
0 & 1 & 0
\end{array}\right] .
$$

The choice of $B_{1}$ indicates that the dynamic coupling signal $h_{2}$ will be applied to the first equation of the slave Rössler system and the choice of $\boldsymbol{B}_{2}$ follows from the assumption that only the variables $x_{2 m}$ and $x_{2 s}$ are measured.

Then, the equations describing a pair of identical Rössler systems interacting via the proposed master-slave configuration with dynamic coupling take the form

$$
\begin{aligned}
& \text { Master: }\left\{\begin{array}{l}
\dot{x}_{1 m}=-x_{2 m}-x_{3 m}, \\
\dot{x}_{2 m}=x_{1 m}+a x_{2 m}, \\
\dot{x}_{3 m}=b+x_{1 m} x_{3 m}-c x_{3 m},
\end{array}\right. \\
& \text { Slave: }\left\{\begin{array}{l}
\dot{x}_{1 s}=-x_{2 s}-x_{3 s}-h_{2}, \\
\dot{x}_{2 s}=x_{1 s}+a x_{2 s}, \\
\dot{x}_{3 s}=b+x_{1 s} x_{3 s}-c x_{3 s},
\end{array}\right. \\
& \text { Dynamic }\left\{\begin{array}{l}
\dot{h}_{1}=-\alpha h_{1}+h_{2}, \\
\dot{h}_{2}=-\gamma_{1} h_{1}-\gamma_{2} h_{2}-k\left(x_{2 m}-x_{2 s}\right) .
\end{array}\right.
\end{aligned}
$$

Let the synchronization errors be defined by $e_{j}=x_{j m}-x_{j s}$, $j=1-3$, and $e_{4}=h_{1}, e_{5}=h_{2}$. Then, the error dynamics is given by

$$
\begin{aligned}
& \dot{e}_{1}=-\left(e_{2}+e_{3}\right)+e_{5}, \\
& \dot{e}_{2}=e_{1}+a e_{2}, \\
& \dot{e}_{3}=x_{1 m} x_{3 m}-x_{1 s} x_{3 s}-c e_{3}, \\
& \dot{e}_{4}=-\alpha e_{4}+e_{5}, \\
& \dot{e}_{5}=-\gamma_{1} e_{4}-\gamma_{2} e_{5}-k e_{2} .
\end{aligned}
$$

Note that Eq. (28) can be written in the form of Eq. (9) with

$$
A_{p}=\left[\begin{array}{ccccc}
0 & -1 & -1 & 0 & 1 \\
1 & a & 0 & 0 & 0 \\
0 & 0 & -c & 0 & 0 \\
0 & 0 & 0 & -\alpha & 1 \\
0 & -k & 0 & -\gamma_{1} & -\gamma_{2}
\end{array}\right],
$$

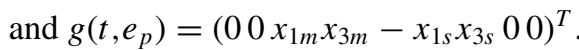

The characteristic polynomial of Eq. (29) is given by

$$
\begin{aligned}
p(\lambda): & =\operatorname{det}(\lambda I-A) \\
& =(\lambda+c)\left(\lambda^{4}+a_{3} \lambda^{3}+a_{2} \lambda^{2}+a_{1} \lambda+a_{0}\right)=0,
\end{aligned}
$$

where

$$
\begin{aligned}
& a_{3}=\alpha-a+\gamma_{2}, \\
& a_{2}=\gamma_{1}-a \alpha-a \gamma_{2}+\alpha g_{2}+1, \\
& a_{1}=\left(\alpha+\gamma_{2}+k-a \gamma_{1}-a \alpha \gamma_{2}\right), \\
& a_{0}=\gamma_{1}+\alpha \gamma_{2}+\alpha k .
\end{aligned}
$$

According to the Ruth-Hurwitz stability test, cf. Ref. [20], the characteristic polynomial Eq. (30) is stable, i.e., all its roots have negative real parts if and only if the following conditions are satisfied:

$$
\begin{aligned}
a_{0}, a_{3} & >0, \\
a_{3} a_{2}-a_{4} a_{1} & >0, \\
a_{3} a_{2} a_{1}-a_{4} a_{1}^{2}-a_{3}^{2} a_{0} & >0 .
\end{aligned}
$$

If we choose

$$
\alpha=a, \quad \gamma_{1}=k, \quad \gamma_{2}=k,
$$

then the above conditions reduce to

$$
\begin{aligned}
k^{2}+(a-1) k-a & >0, \\
b_{3} k^{3}+b_{2} k^{2}+b_{1} k+b_{0} & >0,
\end{aligned}
$$

where $\quad b_{3}=1-a^{2}-3 a, b_{2}=4 a-a^{3}-2, b_{1}=\left(a^{3}+\right.$ $\left.2 a^{2}-3 a\right), b_{0}=-a^{2}$.

Consequently, if the parameter values in the dynamic coupling are chosen as in Eq. (31) and the coupling strength $k$ is chosen such that conditions (32) and (33) are satisfied, then matrix $A_{p}$ in (29) is Hurwitz. Note, however, that choice (31) is not unique. Obviously, there exist other possible choices.

Next, the (local) stability of the synchronous solution $\boldsymbol{x}_{m}=$ $\boldsymbol{x}_{s}$ in the coupled Rössler systems (25)-(27) is investigated-as a function of the coupling strength $k$-by looking at the largest transverse Lyapunov exponent $\lambda_{\max }^{\perp}$.

For the case of the proposed configuration, $\lambda_{\max }^{\perp}$ is determined from Eq. (7), whereas for the traditional master-slave configuration, $\lambda \stackrel{\perp}{\max }$ is computed from $\dot{\xi}=\left[\boldsymbol{D} \boldsymbol{F}\left(\boldsymbol{x}_{m}\right)-k \boldsymbol{C}\right] \xi$, see e.g., Ref. [13]. As mentioned before, the synchronous solution is (locally) stable for $\lambda \lambda_{\max }^{\perp}<0$ and unstable for $\lambda \stackrel{\perp}{\max }>0$. 


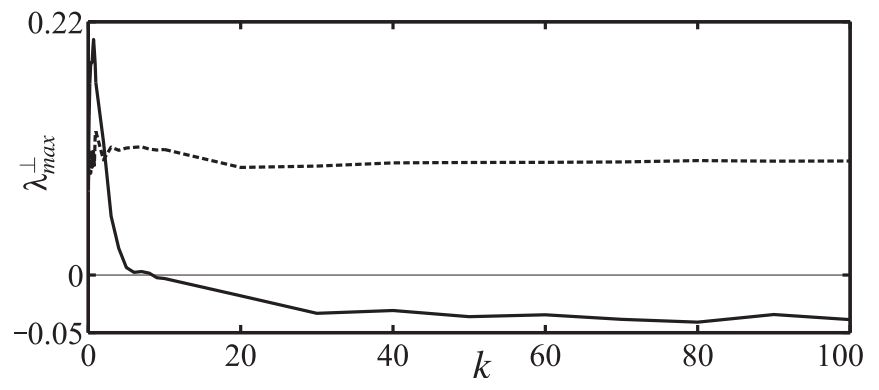

FIG. 2. The largest transverse Lyapunov exponent $\lambda_{\max }^{\perp}$ computed as a function of the coupling strength $k$. The solid line indicates $\lambda_{\max }^{\perp}$ for the proposed coupling scheme, whereas the dashed line shows $\lambda \max _{\max }^{\perp}$ for the standard master-slave configuration. Clearly, the proposed configuration induces synchronization in the slave system for $k>8.4$, whereas for the standard configuration $\lambda_{\max }^{\perp}$ stays positive for all $k>0$ and consequently, the synchronous solution is always unstable.

The obtained results for both configurations are shown in Fig. 2. The solid line shows $\lambda \stackrel{\perp}{\max }$ for the proposed coupling scheme, whereas the dashed line shows $\lambda_{\max }^{\perp}$ for the traditional master-slave configuration. The figure clearly shows that the proposed coupling scheme induces synchronization for $k>8.4$. This is in clear contrast to the standard configuration for which $\lambda \stackrel{\perp}{\max }$ stays always positive, and therefore, the synchronous solution is unstable. In the computations, a modified version of the algorithm of Wolf et al. [21] has been used, and the following parameter values have been considered [19]: $a=0.2, b=0.2, c=5.7$, and $\alpha, \gamma_{1}$, and $\gamma_{2}$ as given in (31).

The above example illustrates the point: By letting the master and slave systems interact through a dynamic coupling, it is possible to synchronize systems that are nonsynchronizable when using the standard master-slave configuration.

Additionally, we illustrate how the proposed coupling removes another limitation of the traditional master-slave scheme. Consider again the Rössler system discussed before, but now assume that the coupling matrix $\boldsymbol{C}$ of the standard configuration, see Eqs. (1) and (2), is defined by $\boldsymbol{C}_{i j}=1$ for $i=j=1$ and $\boldsymbol{C}_{i j}=0$ otherwise. In this case, as shown in Ref. [12], it is possible to induce synchronization in the coupled Rössler systems but only within the narrow interval of coupling strength values satisfying $0.19<k<4.84$ as illustrated in Fig. 3 (dashed line).

On the other hand, consider the proposed coupling scheme (3)-(5) with the coupling matrices,

$$
\boldsymbol{B}_{1}=\left[\begin{array}{ll}
0 & 1 \\
0 & 0 \\
0 & 0
\end{array}\right], \quad \boldsymbol{B}_{2}=\left[\begin{array}{lll}
0 & 0 & 0 \\
1 & 0 & 0
\end{array}\right],
$$

and take the gains $\alpha, \gamma_{1}$, and $\gamma_{2}$ as given in (31).

Then, synchronization is observed for $k>0.45$ as shown in Fig. 3 (solid line). This result clearly shows that, if the interaction between the master and the slave is dynamic, then the value of the coupling strength $k$ can be arbitrarily large.

The results presented in Fig. 3 can be further explained by looking at the synchronization error dynamics. For the case of the classical master slave configuration, the synchronization

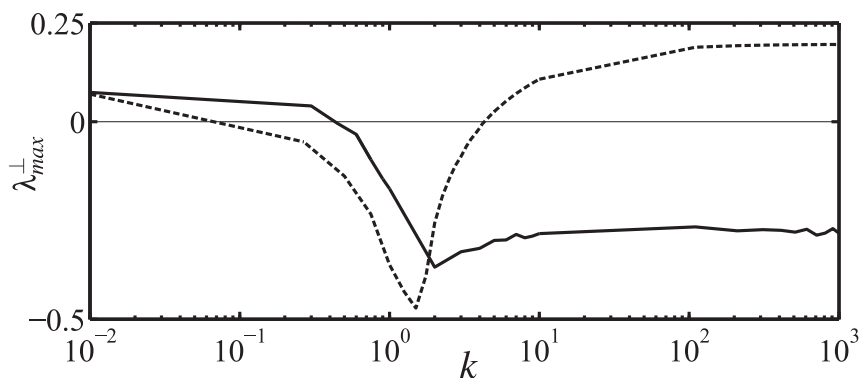

FIG. 3. $\lambda_{\max }^{\perp}$ computed as a function of the coupling strength $k$ (on the logarithmic scale). The proposed configuration successfully extends the onset of synchronization beyond the narrow interval of the standard configuration. The solid and dashed lines have the same meaning as in Fig. 2.

error dynamics can be written in the form of Eq. (9) with

$$
\boldsymbol{A}_{p}=\left[\begin{array}{ccc}
-k & -1 & -1 \\
1 & a & 0 \\
0 & 0 & -c
\end{array}\right],
$$

and $\boldsymbol{g}_{p}\left(t, \boldsymbol{e}_{p}\right)=\boldsymbol{f}\left(\boldsymbol{x}_{m}\right)-\boldsymbol{f}\left(\boldsymbol{x}_{s}\right)$. The matrix given in Eq. (35) has the characteristic polynomial,

$$
\begin{aligned}
p_{c}(\lambda): & =\left(\lambda I-\boldsymbol{A}_{p}\right) \\
& =(\lambda+c)\left[\lambda^{2}+(k-a) \lambda-a k+1\right] .
\end{aligned}
$$

Since $c$ is a positive parameter, a sufficient and necessary condition for the stability of (36) - in terms of the coupling strength-is

$$
a<k<1 / a \text {. }
$$

In contrast, for the proposed master-slave configuration, the obtained $\boldsymbol{A}_{p}$ matrix of the error dynamics, see Eq. (10), is given by

$$
\boldsymbol{A}_{p}=\left[\begin{array}{ccccc}
0 & -1 & -1 & 0 & 1 \\
1 & a & 0 & 0 & 0 \\
0 & 0 & -c & 0 & 0 \\
0 & 0 & 0 & -\alpha & 1 \\
-k & 0 & 0 & -\gamma_{1} & -\gamma_{2}
\end{array}\right]
$$

The characteristic polynomial of this matrix is

$$
\begin{aligned}
p(\lambda): & =\operatorname{det}\left(\lambda I-\boldsymbol{A}_{p}\right) \\
& =(\lambda+c)\left(a_{4} \lambda^{4}+a_{3} \lambda^{3}+a_{2} \lambda^{2}+a_{1} \lambda+a_{0}\right)=0,
\end{aligned}
$$

where $a_{4}=1, a_{3}=\alpha-a+\gamma_{2}, a_{2}=\gamma_{1}+k-\alpha a+\alpha \gamma_{2}-$ $a \gamma_{2}+1, a_{1}=\alpha+\gamma_{2}+\alpha k-a \gamma_{1}-a k-\alpha a \gamma_{2}$, and $a_{0}=$ $\gamma_{1}+\alpha \gamma_{2}-\alpha a k$. If the parameters $\alpha, \gamma_{1}$, and $\gamma_{2}$ are chosen as in Eq. (31), then the characteristic polynomial given in Eq. (39) is stable, i.e., all its roots have negative real parts if and only if the following conditions are satisfied:

$$
\begin{aligned}
k^{2}+ & \frac{1}{2}\left(a^{2}+1\right) k+\frac{a}{2}(a-1)>0, \\
b_{3} k^{3}+b_{2} k^{2}+b_{1} k+b_{0} & >0,
\end{aligned}
$$

where $b_{3}=-2 a^{2}-4 a+3, b_{2}=(a+2)\left(1-a^{3}-a\right), b_{1}=$ $-a\left(-2 a^{3}-a^{2}+5 a-2\right), b_{0}=-a^{4}+3 a^{2}-2 a$. 

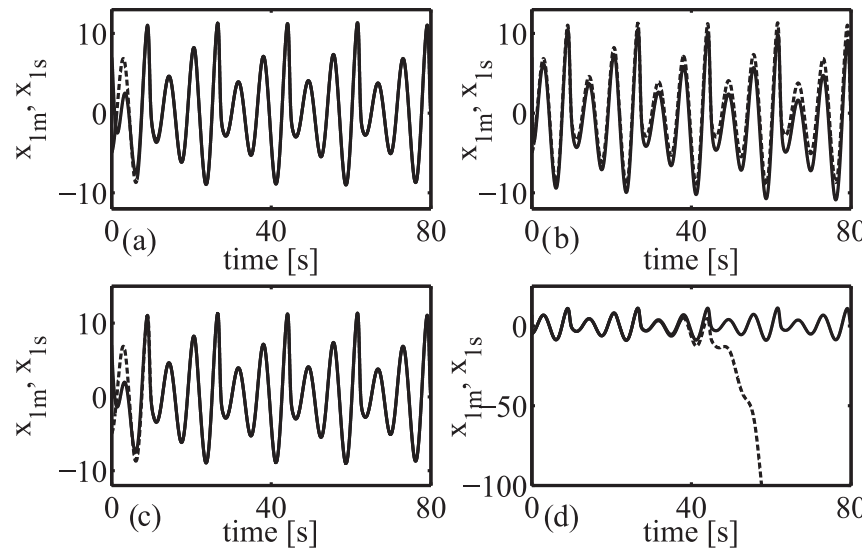

FIG. 4. Representative time series for two coupled Rössler systems for different coupling strengths. Left: Proposed coupling scheme. Right: Classical master-slave configuration. For (a) and (b): $k=5$. For (c) and (d): $k=1000$. The proposed coupling scheme induces synchronization, whereas the classical master-slave approach fails to synchronize them. $x_{1 m}$ : solid; $x_{1 s}$ : dashed.

From a quick comparison between condition (37) for the classical scheme and conditions (40) and (41) for the proposed scheme, it is possible to reach to the following conclusion: In the classical scheme, the matrix $\boldsymbol{A}_{p}$ of the error dynamics can be converted into a Hurwitz matrix in a narrow interval of coupling strength values as shown in Eq. (37). On the other hand, for the proposed scheme, the corresponding $\boldsymbol{A}_{p}$ matrix is converted into a Hurwitz matrix for sufficiently large coupling strength values as clearly seen from conditions (40) and (41).

This argument is further supported by replacing the value of $a=0.2$ as used in Fig. 3 into conditions (37), (40), and (41). For the aforementioned value of parameter $a$, which indeed is an intrinsic parameter of the Rössler system, condition (37) becomes $0.2<k<5$. In contrast, for the proposed scheme, conditions (40) and (41) reduce to $k>0.37$. These bounds are in good agreement with the bounds observed in Fig. 3.

Additionally, some representative time series are presented in Fig. 4. The figure shows the time series of two coupled Rössler systems with coupling matrices as given in (34), parameter values as used before, and different values of coupling strength. In particular, the plots on the left show the obtained results with the proposed dynamic master-slave configuration, whereas the plots on the right correspond to the traditional master-slave coupling. In plots (a) and (b) the coupling strength has been set to $k=5$, whereas for plots (c) and (d), the coupling strength has been set to $k=1000$. For both values of $k$, the proposed dynamic master-slave configuration achieves synchronization.

On the other hand, for the considered values of $k$, the classical configuration fails to synchronize the slave and moreover, for $k=1000$, the trajectories of the slave system diverge.

At this point, it is important to mention that in the previous analysis, we have considered $h_{2}$ as the coupling signal. However, similar results can be obtained if instead, the coupling signal $h_{1}$ is used to synchronize the slave to the master.

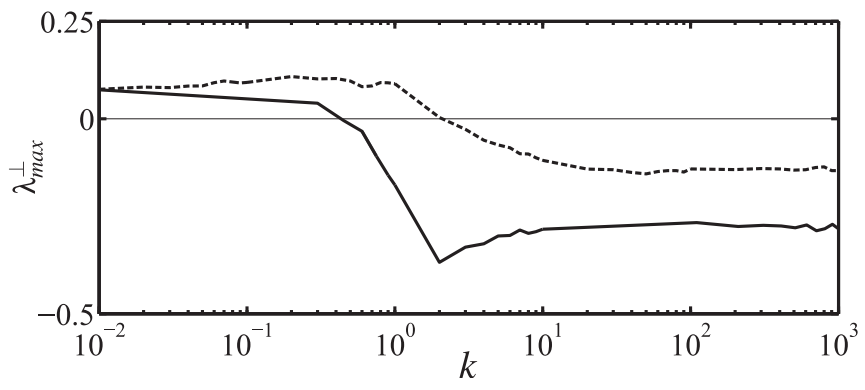

FIG. 5. Largest transverse Lyapunov exponent $\lambda_{\max }^{\perp}$ as a function of the coupling strength $k$. The dashed line: $\lambda_{\max }^{\perp}$ when $h_{1}$ is used as the coupling signal. The solid line: $\lambda_{\max }^{\perp}$ when the coupling signal is $h_{2}$. In both cases, synchronization is achieved for large $k$. However, a smaller coupling strength is required when the coupling signal $h_{2}$ is used.

In this case, the coupling matrix $B_{1}$ given in (34) should be modified to

$$
B_{1}=\left[\begin{array}{ll}
1 & 0 \\
0 & 0 \\
0 & 0
\end{array}\right] \text {. }
$$

Again, the onset of stable synchronous motion when $h_{1}$ is used as the coupling variable can be investigated as a function of the coupling strength $k$ by looking at the largest (transverse) Lyapunov exponent computed from the variational equation (7) with $B_{1}$ as given in (42). In the computations, the parameter values are exactly the same as those used in the previous analysis - where $h_{2}$ was used as the coupling signal. The obtained results are shown in Fig. 5. The dashed line shows $\lambda \stackrel{\perp}{\max }$ for the case when the coupling signal is $h_{1}$. For the sake of easy comparison, Fig. 5 also shows $\lambda \stackrel{\perp}{\max }$ for the case when $h_{2}$ is used as the coupling signal (solid line) as already shown in Fig. 2. It can easily be seen that both coupling signals, either $h_{1}$ or $h_{2}$, extend the onset of synchronization beyond the interval obtained with the classical static master-slave configuration. However, there is a notorious difference, namely, that the threshold value of coupling strength is larger for the case when $h_{1}$ is considered as a coupling signal. Specifically, when $h_{1}$ is used, the slave system synchronizes to the master system for $k>2$, whereas for the case when $h_{2}$ is used, the systems synchronize for $k>0.45$. These results suggest that $h_{2}$ is "the best choice" (at least for the parameter settings considered here) in the sense that a smaller coupling strength is required to synchronize the systems. However, we want to stress the fact that both coupling signals have a very similar performance for large coupling strengths.

A final comment. The previous results have been obtained for a particular choice of $\gamma_{1}$ and $\gamma_{2}$, see Eq. (31). However, as mentioned before, this choice is not unique. Many different choices are possible as long as $\lambda_{\max }^{\perp}$ stays negative. This is illustrated in Fig. 6 where the largest (transverse) Lyapunov exponent is computed as a function of the coupling strength $k$ and parameter $\gamma_{1}$. For the computation, the variational equation (7) is used with coupling matrices $\boldsymbol{B}_{1}$ and $\boldsymbol{B}_{2}$ as given in (24). The parameter values are the same as those used to in the analysis presented in Fig. 2, and $\gamma_{2}$ is as given in (31). From 


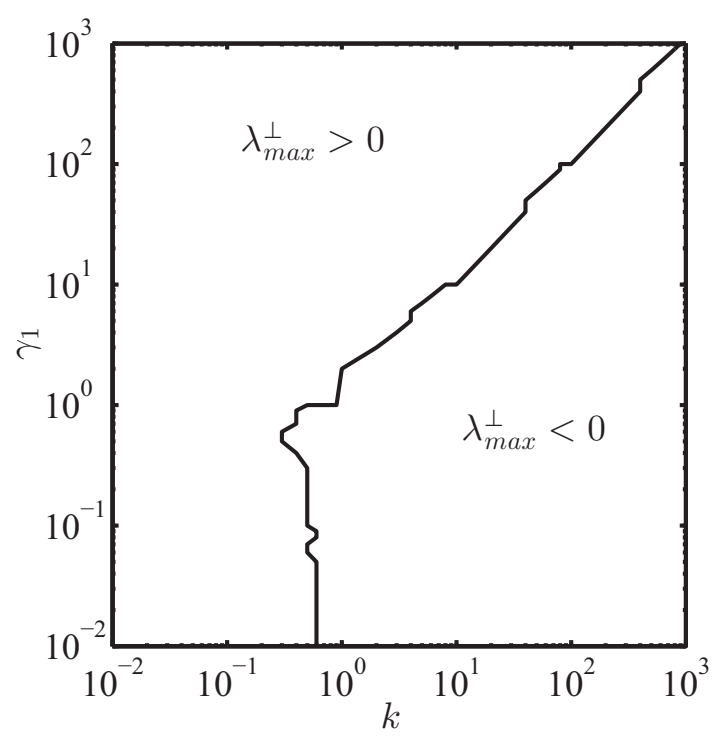

FIG. 6. Largest transverse Lyapunov exponent on the $\left(\gamma_{1}, k\right)$ plane. There exist several values of $\gamma_{1}$ for which synchronization can be observed besides the choice given in Eq. (31).

Fig. 6 it is clear that there exists a large region on the $\left(k, \gamma_{1}\right)$ plane where $\lambda_{\max }^{\perp}<0$.

\section{Example 3: Application to typical chaotic systems}

In this section, the proposed coupling scheme is applied to different chaotic systems for which a traditional master-slave configuration induces synchronization only within a narrow interval of coupling strength values. Specifically, the following chaotic systems are considered: Chua's circuit, the HindmarshRose neuronal model, and the forced van der Pol and Duffing systems. The parameter values for each system are chosen such that chaotic behavior is observed. Moreover, for the case of the traditional master-slave configuration, these systems have been already discussed in Ref. [12].

Table I presents the model for each system, the coupling and Jacobian matrices for each configuration, and the parameter values used in the analysis. For each case, the coupling matrix $C$, corresponding to the traditional master-slave configuration, has been obtained from Ref. [12] where it has been shown that, for this choice of $C$, synchronization is only achieved in a narrow interval of coupling strength values.

The local stability of the synchronous solution is investigated by looking at the largest transverse Lyapunov exponent-denoted by $\lambda_{\max }^{T}$-which is computed from the corresponding variational equation. For the case of the classical master-slave configuration, the variational equation is given by $\dot{\boldsymbol{\xi}}=\left[\boldsymbol{D} \boldsymbol{F}\left(\boldsymbol{x}_{m}\right)-k \boldsymbol{C}\right] \boldsymbol{\xi}$, whereas for the proposed coupling scheme, the variational equation is given in Eq. (7). For each system, the Jacobian matrices corresponding to the traditional and the proposed coupling configurations are given in the middle and right columns, respectively, of Table I.

Figure 7 shows the largest transverse Lyapunov exponent $\lambda_{\max }^{\perp}$, computed as a function of the coupling strength, for different systems: (a) Chua's circuit, (b) The Hindmarsh-Rose model, (c) the forced van der Pol system, and (d) the forced
Duffing equation. The details for each system are summarized in Table I.

The obtained results reveal that the proposed master-slave configuration with dynamic interaction may induce synchronization in several chaotic systems for arbitrarily large coupling strength values. These results are in clear contrast to the classical master-slave configuration where synchronization is observed in a limited interval of coupling strength values.

\section{The dimension of the dynamic interconnection influences the onset of synchronization}

So far, the obtained results suggest that a second order dynamic interconnection enhances the onset of synchronization with respect to a static coupling. A natural question at this point is: Does the order or dimension of the dynamic interconnection have an influence on the onset of synchronization?

To elaborate on this question, we will compare the results obtained above, which correspond to a second order dynamic coupling, to the results obtained when using the first order dynamic interconnection,

$$
\begin{aligned}
\dot{\boldsymbol{x}}_{m} & =\boldsymbol{F}\left(\boldsymbol{x}_{m}\right), \\
\dot{\boldsymbol{x}}_{s} & =\boldsymbol{F}\left(\boldsymbol{x}_{s}\right)-\boldsymbol{b}_{1} \eta, \\
\dot{\eta} & =-\gamma \eta-k \boldsymbol{b}_{2}\left(\boldsymbol{x}_{m}-\boldsymbol{x}_{s}\right),
\end{aligned}
$$

where $\boldsymbol{x}_{m}, \boldsymbol{x}_{s}$, and $\boldsymbol{F}$ are as defined before, $\eta \in \mathbb{R}$ is the scalar coupling signal, $\gamma \in \mathbb{R}_{+}$is a constant parameter, and the vectors $\boldsymbol{b}_{1} \in \mathbb{R}^{n}$ and $\boldsymbol{b}_{2} \in \mathbb{R}^{1 \times n}$ are the coupling vectors.

In particular, the examples presented in Secs. III A and III B are revisited. The final aim is to compare the performance of the first order configuration (43)-(45) to the performance obtained when using the second order scheme (3)-(6).

\section{Example 1 revisited}

Consider again Example III A, i.e., a pair of harmonic oscillators. Following the assumptions made for deriving the coupling matrices (15), we obtain the coupling vectors,

$$
\boldsymbol{b}_{1}=\left[\begin{array}{l}
0 \\
1
\end{array}\right], \quad \boldsymbol{b}_{2}=\left[\begin{array}{ll}
1 & 0
\end{array}\right] \text {. }
$$

Consequently, the coupled harmonic oscillators, interacting via the first order dynamic configuration (43)-(45) are written in the form

$$
\begin{aligned}
& \text { Master: }\left\{\begin{array}{l}
\dot{x}_{1 m}=x_{2 m}, \\
\dot{x}_{2 m}=-\omega^{2} x_{1 m},
\end{array}\right. \\
& \text { Slave: }\left\{\begin{array}{l}
\dot{x}_{1 s}=x_{2 s}, \\
\dot{x}_{2 s}=-\omega^{2} x_{1 s}-\eta,
\end{array}\right. \\
& \text { Dynamic }\left\{\dot{\eta}=-\gamma \eta-k\left(x_{1 m}-x_{1 s}\right)\right. \text {. }
\end{aligned}
$$

The stability of the synchronous solution in the coupled systems (48) and (49) is determined from the error dynamics $\dot{\boldsymbol{e}}_{p}=\boldsymbol{A}_{p} \boldsymbol{e}_{p}$, where $\boldsymbol{e}_{p}=\left(e_{1}, e_{2}, \eta\right)^{T}, e_{1}=x_{1 m}-x_{1 s}$, 
TABLE I. Classical vs proposed master-slave scheme for different dynamical systems.

\begin{tabular}{|c|c|c|}
\hline System & Classical master-slave coupling & Proposed coupling \\
\hline $\begin{array}{l}\text { Chua's system [22]: } \\
\qquad \begin{aligned} \dot{x} & =\sigma[y-x-\phi(x)] \\
\dot{y} & =x-y+z \\
\dot{z} & =-\beta y\end{aligned} \\
\text { with } \phi(x)=b x+\frac{1}{2}(a-b)(|x+1|-|x-1|),\end{array}$ & $\begin{array}{c}\text { Jacobian matrix: } \\
{\left[\boldsymbol{D F}\left(\boldsymbol{x}_{m}\right)-k \boldsymbol{C}\right]=\left(\begin{array}{ccc}-\sigma-\sigma p(x) & \sigma & 0 \\
1 & -1 & 1 \\
0 & -\beta & -k\end{array}\right)}\end{array}$ & $\begin{array}{c}\text { Jacobian matrix: } \\
\widetilde{\boldsymbol{D F}}\left(\boldsymbol{x}_{m}\right)=\left(\begin{array}{ccccc}-\sigma-\sigma p(x) & \sigma & 0 & 0 & 0 \\
1 & -1 & 1 & 0 & 0 \\
0 & -\beta & 0 & 1 & 0 \\
0 & 0 & 0 & -\alpha & 1 \\
0 & 0 & -k & -\gamma_{1} & -\gamma_{2}\end{array}\right),\end{array}$ \\
\hline $\begin{array}{l}\text { Parameter values: } \\
\sigma=10, \beta=14.87, a=-1.27, b=-0.68,\end{array}$ & $\begin{array}{c}p(x)=\frac{1}{2}(b \operatorname{sign}(|x|-1)+b)-\frac{1}{2}(a \operatorname{sign}(|x|-1)-a), \\
\text { Coupling matrix: } \\
C=\left[\begin{array}{lll}0 & 0 & 0 \\
0 & 0 & 0 \\
0 & 0 & 1\end{array}\right],\end{array}$ & $\begin{array}{l}\alpha=1, \gamma_{1}=k, \gamma_{2}=\frac{k}{5} \\
\text { Coupling matrices: } \\
B_{1}=\left[\begin{array}{ll}0 & 0 \\
0 & 0 \\
1 & 0\end{array}\right], B_{2}=B_{1}^{T}\end{array}$ \\
\hline Hindmarsh-Rose (neuron) model [23]: & Jacobian matrix: & Jacobian matrix: \\
\hline $\begin{array}{l}\dot{x}=y+3 x^{2}-x^{3}-z+I \\
\dot{y}=1-5 x^{2}-y \\
\dot{z}=-r z+r s(x+p)\end{array}$ & {$\left[\boldsymbol{D F}\left(\boldsymbol{x}_{m}\right)-k \boldsymbol{C}\right]=\left[\begin{array}{ccc}6 x-3 x^{2} & 1-k-1 \\
-10 x & -1 & 0 \\
r s & 0 & -r\end{array}\right]$} & $\begin{array}{l}\widetilde{\boldsymbol{D F}}\left(\boldsymbol{x}_{m}\right)=\left[\begin{array}{ccccc}6 x-3 x^{2} & 1 & -1 & 0 & 1 \\
-10 x & -1 & 0 & 0 & 0 \\
r s & 0 & -r & 0 & 0 \\
0 & 0 & 0 & -\alpha & 1 \\
0 & -k & 0 & -\gamma_{1} & -\gamma_{2}\end{array}\right], \\
\alpha=1, \gamma_{1}=1, \gamma_{2}=k\end{array}$ \\
\hline $\begin{array}{l}\text { Parameter values: } \\
I=3.2, r=0.006, s=4, p=1.6\end{array}$ & $\begin{array}{l}\text { Coupling matrix: } \\
\qquad C=\left[\begin{array}{lll}0 & 1 & 0 \\
0 & 0 & 0 \\
0 & 0 & 0\end{array}\right]\end{array}$ & $\begin{array}{c}\text { Coupling matrices: } \\
B_{1}=\left[\begin{array}{ll}0 & 1 \\
0 & 0 \\
0 & 0\end{array}\right], B_{2}=\left[\begin{array}{lll}0 & 0 & 0 \\
0 & 1 & 0\end{array}\right]\end{array}$ \\
\hline van der Pol equation [24]: & Jacobian matrix: & Jacobian matrix: \\
\hline $\begin{array}{l}\dot{x}=y, \\
\dot{y}=-x+d\left(1-x^{2}\right) y+a \sin \eta t,\end{array}$ & {$\left[\boldsymbol{D F}\left(\boldsymbol{x}_{m}\right)-k \boldsymbol{C}\right]=\left[\begin{array}{cc}0 & 1-k \\
-1-2 d x y & d\left(1-x^{2}\right)\end{array}\right]$} & $\begin{array}{l}\widetilde{\boldsymbol{D F}}\left(\boldsymbol{x}_{m}\right)=\left[\begin{array}{cccc}0 & 1 & 0 & 1 \\
-1-2 d x y & d\left(1-x^{2}\right) & 0 & 0 \\
0 & 0 & -\alpha & 1 \\
0 & -k & -\gamma_{1} & -\gamma_{2}\end{array}\right. \\
\alpha=1, \gamma_{1}=1, \gamma_{2}=10 k .\end{array}$ \\
\hline $\begin{array}{l}\text { Parameter values: } \\
\eta=4.065, d=3, a=15,\end{array}$ & $\begin{array}{l}\text { Coupling matrix: } \\
\qquad C=\left[\begin{array}{ll}0 & 1 \\
0 & 0\end{array}\right]\end{array}$ & $\begin{array}{c}\text { Coupling matrices: } \\
B_{1}=\left[\begin{array}{ll}0 & 1 \\
0 & 0\end{array}\right], B_{2}=\left[\begin{array}{ll}0 & 0 \\
0 & 1\end{array}\right]\end{array}$ \\
\hline Forced Duffing equation [25]: & Jacobian matrix: & Jacobian matrix: \\
\hline $\begin{array}{l}\dot{x}=y \\
\dot{y}=-\delta y-x^{3}+q \sin \eta t\end{array}$ & {$\left[\boldsymbol{D F}\left(\boldsymbol{x}_{m}\right)-k \boldsymbol{C}\right]=\left[\begin{array}{cc}0 & 1-k \\
-3 x^{2} & -\delta\end{array}\right]$} & $\begin{array}{l}\widetilde{\boldsymbol{D F}}\left(\boldsymbol{x}_{m}\right)=\left[\begin{array}{cccc}0 & 1 & 0 & 1 \\
-3 x^{2} & -\delta & 0 & 0 \\
0 & 0 & -\alpha & 1 \\
0 & -k & -\gamma_{1} & -\gamma_{2}\end{array}\right] \\
\alpha=1, \gamma_{1}=1, \gamma_{2}=k\end{array}$ \\
\hline $\begin{array}{l}\text { Parameter values: } \\
\eta=1, \delta=0.1, q=5.6\end{array}$ & $\begin{array}{l}\text { Coupling matrix: } \\
\qquad C=\left[\begin{array}{ll}0 & 1 \\
0 & 0\end{array}\right]\end{array}$ & $\begin{array}{l}\text { Coupling matrices: } \\
B_{1}=\left[\begin{array}{ll}0 & 1 \\
0 & 0\end{array}\right], B_{2}=\left[\begin{array}{ll}0 & 0 \\
0 & 1\end{array}\right]\end{array}$ \\
\hline
\end{tabular}

$e_{2}=x_{2 m}-x_{2 s}$ and

$$
\boldsymbol{A}_{p}=\left[\begin{array}{ccc}
0 & 1 & 0 \\
-\omega^{2} & 0 & 1 \\
-k & 0 & -\gamma
\end{array}\right] .
$$

This matrix is Hurwitz if and only if

$$
-\gamma \omega^{2}<k<0
$$

From this result, and the result obtained in Sec. III A, the following is clear: If the dimension of the dynamic interconnection is one, synchronization is induced for a bounded interval of negative coupling strengths as shown in Eq. (51). In contrast, if the oscillators interact through a second order coupling, then synchronization can be achieved for any positive coupling strength, i.e., for $k>0$, see below Eq. (21). Although in both cases synchronization is achieved, the second order scheme seems to have a better performance in the sense that, for a fixed set of parameters, this scheme allows a larger interval of coupling strength values for which synchronization is achieved.

\section{Example 2 revisited}

Now, consider again the pair of chaotic Rössler systems as discussed in Example III B. The same assumptions used for deriving the coupling matrices (24) yield to the following coupling vectors:

$$
\boldsymbol{b}_{1}=\left[\begin{array}{l}
1 \\
0 \\
0
\end{array}\right], \quad \boldsymbol{b}_{2}=\left[\begin{array}{lll}
0 & 1 & 0
\end{array}\right] .
$$

Next, the local stability of the synchronous solution in the coupled Rössler systems with first order dynamic coupling is investigated by computing $\lambda_{\max }^{\perp}$ as a function of $k$ from the variational equation (7) in which the vector $\boldsymbol{h}$ should be replaced by the scalar $\eta$, the coupling matrices $\boldsymbol{B}_{1}$ and $\boldsymbol{B}_{2}$ should be replaced by the coupling vectors $\boldsymbol{b}_{1}$ and $\boldsymbol{b}_{2}$ as defined above, and finally, matrix $\boldsymbol{G}$ is replaced by the scalar $\gamma$. The parameter values are as used in Sec. III A and $\gamma=1$.

The obtained results are depicted in Fig. 8. Clearly, $\lambda \underset{\max }{\perp}<0$ in the narrow interval of negative coupling strength values 

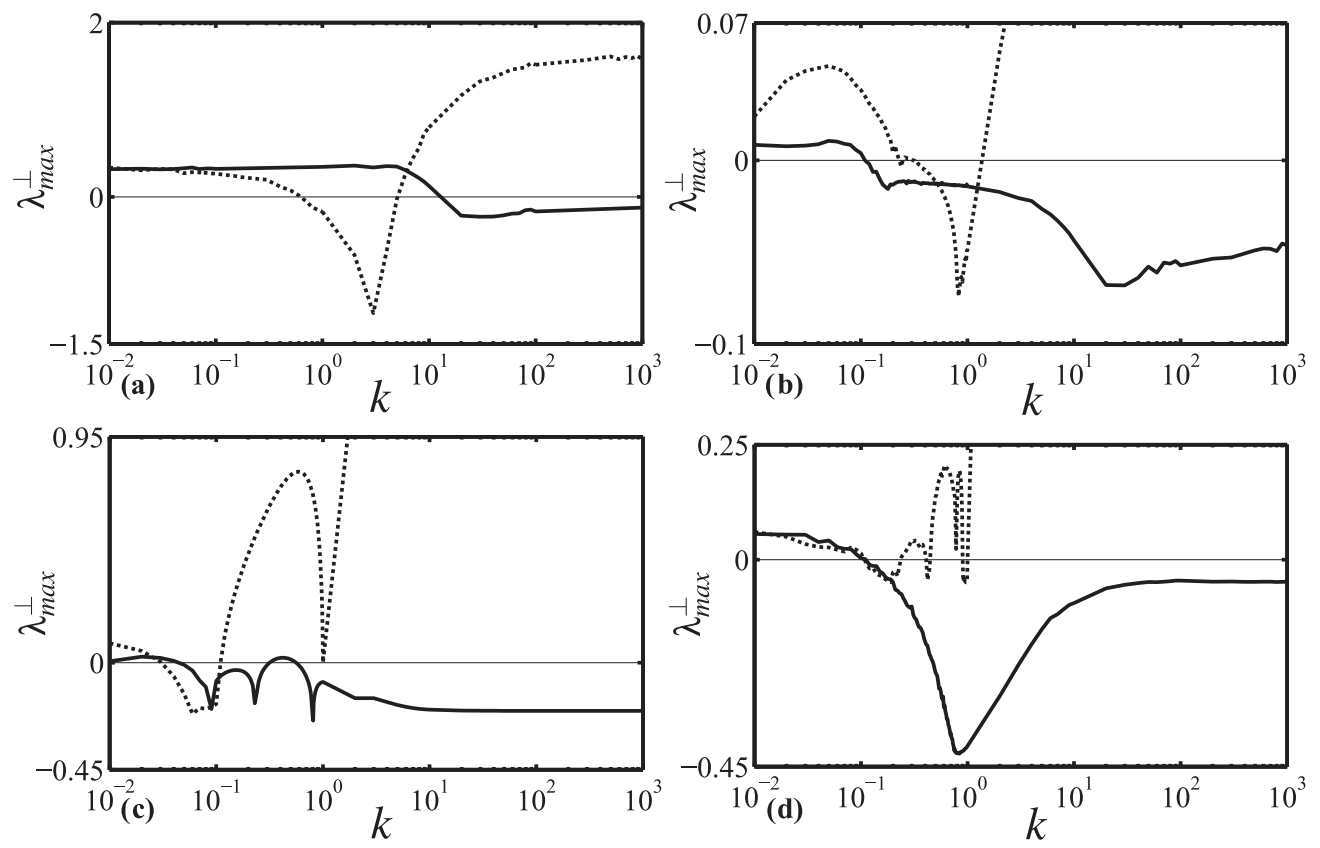

FIG. 7. Largest transverse Lyapunov exponent $\lambda_{\max }^{\perp}$ for different dynamical systems computed as a function of the coupling strength $k$ (on the logarithmic scale). (a) Chua's circuit. (b) The Hindmarsh-Rose neuronal model. (c) The forced van der Pol system. (d) The forced Duffing equation. The details for each system are given in Table I. In all panels, the dashed lines show $\lambda_{\max }^{\perp}$ for the traditional (static) configuration, whereas the solid lines denote $\lambda_{\max }^{\perp}$ for the proposed configuration. In all cases, the proposed configuration extends the onset of synchronization beyond the narrow interval observed in the classical configuration.

$-0.92<k<-0.37$. In contrast, for the second order case, it has been found that $\lambda_{\max }<0$ for $k>8.4$, as shown in Fig. 2 (solid line). Hence, the second order dynamic coupling "facilitates" the onset of synchronization: There exists a larger interval of coupling strengths, compared to the first order case, for which synchronization is achieved.

Table II summarizes the obtained results. From this table, it becomes evident that the order of the dynamic interconnection has an influence on the onset of synchronization.

The results shown in Table II indicate that for a second order dynamic coupling, the interval of coupling strength values, for which synchronization is observed, is larger than the interval obtained with a first order dynamic coupling. This difference is explained as follows. For the first order scheme given in Eqs. (43)-(45), the resulting matrix $\boldsymbol{A}_{p}$ of the synchronization error dynamics, see Eq. (9), is Hurwitz only within a narrow

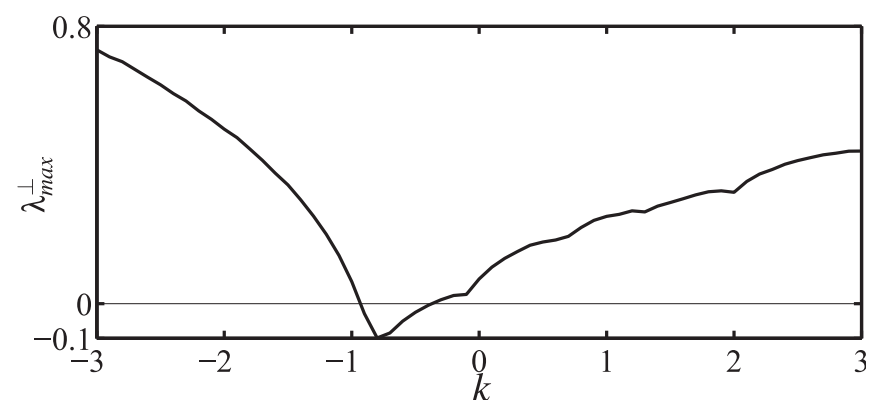

FIG. 8. $\lambda_{\max }^{\perp}$ as a function of $k$ for the first order dynamic master-slave configuration (43)-(45). Synchronization can possibly be achieved in the interval $-0.92<k<-0.37$. Compare to Fig. 2 (solid line). interval of coupling strength values $k$. In contrast, for the second order scheme given in Eqs. (3)-(5), the obtained matrix $\boldsymbol{A}_{p}$ can be converted into a Hurwitz matrix for arbitrarily large values of coupling strength $k$ by a suitable choice of the control parameters $\alpha, \gamma_{1}$, and $\gamma_{2}$, which ultimately results in the local stability of the synchronous solution.

\section{DISCUSSION AND CONCLUSIONS}

The research presented here provides a solution to the problem of synchronizing dynamical systems that are nonsynchronizable with a standard master-slave coupling. The key element in the new scheme is the dynamic coupling, through which the slave system interacts with the master.

Note, however, that we are not claiming that the proposed synchronization scheme has a universal applicability. Instead,

TABLE II. Values of coupling strength $k$ for which synchronization is achieved. The static master-slave scheme fails to achieve synchronization. On the other hand, the first order coupling induces synchronization only for a bounded interval of negative coupling strengths, whereas for a second order coupling synchronization is achieved for any $k$ satisfying $k>k_{c}$, where $k_{c} \geqslant 0$ is a threshold or critical value.

\begin{tabular}{lccc}
\hline \hline & $\begin{array}{c}\text { Static } \\
\text { coupling } \\
\text { Eqs. (1) and (2) }\end{array}$ & $\begin{array}{c}\text { Dynamic coupling } \\
\text { first order } \\
\text { Eqs. (43)-(45) }\end{array}$ & $\begin{array}{c}\text { Dynamic coupling } \\
\text { second order } \\
\text { Eqs. (3)-(5) }\end{array}$ \\
\hline $\begin{array}{l}\text { Harmonic } \\
\text { oscillator }\end{array}$ & None & $-\alpha \omega^{2}<k<0$ & $k>0$ \\
$\begin{array}{l}\text { Rössler } \\
\text { system }\end{array}$ & None & $-0.92<k<-0.37$ & $k>8.4$ \\
\hline
\end{tabular}


we want to convey the message that a dynamic interconnection may enhance the onset of synchronization in certain cases where a static coupling fails. So far, we have realized that the proposed scheme will work for the cases where a suitable value for the parameters $\alpha, \gamma_{1}$, and $\gamma_{2}$ in the dynamic coupling (5) and (6) can be found such that matrix $\boldsymbol{A}_{p}$ in Eq. (9) is converted into a Hurwitz matrix.

In other cases, the dynamic master-slave scheme presented here allows for inducing synchronization for arbitrarily large coupling strength values in systems for which the standard coupling has an upper bound on the value of coupling strength. At first sight, this improvement may seem artificial. However, we want to stress the fact that there exist cases where a large coupling strength value is indeed required. This is often the case in, for example, physical implementations where the unavoidable (small) mismatches between systems may be compensated by a large coupling strength to enforce synchronization [26].

In contrast to Ref. [7] where synchronization is achieved by transiently uncoupling the slave from the master, in the proposed configuration, the coupling is dynamic, it is always active, and it vanishes once the systems are synchronized.

The reader should realize that further work is still needed. For example, it is necessary to derive a systematic and rigorous method for computing the "optimal" values of the constants $\alpha, \gamma_{1}$, and $\gamma_{2}$ in the dynamic coupling, see Eq. (6). Another possible extension of this paper is to consider more than one slave system dynamically connected to a master system through a common coupling signal, i.e., a network of slave systems with dynamic interconnections.
[1] L. M. Pecora and T. L. Carroll, Phys. Rev. Lett. 64, 821 (1990).

[2] R. Brown, N. F. Rulkov, and E. R. Tracy, Phys. Rev. E 49, 3784 (1994).

[3] H. Fujisaka and T. Yamada, Prog. Theor. Phys. 69, 32 (1983).

[4] V. S. Afraimovich, N. N. Verichev, and M. I. Rabinovich, Radiophys. Quantum Electron. 29, 795 (1986).

[5] G. Katriel, Phys. D 237, 2933 (2008).

[6] J. A. K. Suykens, P. F. Curran, and L. O. Chua, Int. J. Bifurcation Chaos 07, 671 (1997).

[7] M. Schröder, M. Mannattil, D. Dutta, S. Chakraborty, and M. Timme, Phys. Rev. Lett. 115, 054101 (2015).

[8] C. Tresser, P. A. Worfolk, and H. Bass, Chaos 5, 693 (1995).

[9] C. Li, W. Sun, and J. Kurths, Phys. Rev. E 76, 046204 (2007).

[10] H. Nijmeijer and A. Rodriguez-Angeles, Synchronization of Mechanical Systems, Series on Nonlinear Science Series A Vol. 46 (World Scientific, Singapore, 2003).

[11] A. Y. Pogromsky and H. Nijmeijer, IEEE Trans. Circuits Syst. I Fundam. Theory Appl. 48, 152 (2001).

[12] L. Huang, Q. Chen, Y.-C. Lai, and L. M. Pecora, Phys. Rev. E 80, 036204 (2009).

[13] L. M. Pecora and T. L. Carroll, Phys. Rev. Lett. 80, 2109 (1998).

[14] M. Bennett, M. F. Schatz, H. Rockwood, and K. Wiesenfeld, Proc. R. Soc. London, Ser. A 458, 563 (2002).
[15] J. P. Ramirez, K. Aihara, R. Fey, and H. Nijmeijer, Phys. D 270, 11 (2014).

[16] A. S. Pikovsky, M. G. Rosenblum, and J. Kurths, Synchronization: A Universal Concept in Nonlinear Sciences (Cambridge University Press, Cambridge, UK, 2003).

[17] H. Khalil, Nonlinear Systems, 3rd ed. (Prentice-Hall, Englewood Cliffs, NJ, 2002).

[18] T. Kailath, Linear Systems (Prentice-Hall, Englewood Cliffs, NJ, 1980).

[19] O. Rössler, Phys. Lett. A 57, 397 (1976).

[20] G. Meinsma, Syst. Control Lett. 25, 237 (1995).

[21] A. Wolf, J. B. Swift, H. L. Swinney, and J. A. Vastano, Phys. D 16, 285 (1985).

[22] T. Matsumoto, L. Chua, and M. Komuro, IEEE Trans. Circuit Syst. 32, 797 (1985).

[23] J. L. Hindmarsh and R. M. Rose, Proc. R Soc. London, Ser. B 221, 87 (1984).

[24] R. Mettin, U. Parlitz, and W. Lauterborn, Int. J. Bifurcation Chaos 03, 1529 (1993).

[25] The Duffing Equation: Nonlinear Oscillators and Their Behaviour, edited by I. Kovacic and M. J. Brennan (Wiley, Chichester, UK, 2011).

[26] M. Brede, Eur. Phys. J. B 62, 87 (2008). 